\title{
Design considerations for improved dock mitre gates
}

\section{Cullen}

\section{J. Lewin, Consultant}

Mr Cullen draws justified attention to the design limitations of conventional dock mitre gates. Remedial work or improvements of existing structures is difficult and often impossible. It appears that there is little development of traditional designs to make them more efficient and to overcome the practical limitations of accuracy of construction which can negate design assumptions.

Sealing is a deficient area. At other gate installations, such as spillway or tunnel gates and river control gates, seal leakage can cause gate vibration which has serious consequences. Good seal design and accuracy of installation can overcome the problem, although it is not often achieved in practice.

Mitre gate designers should study how sealing difficulties are solved in related applications. Fig. 3 of Mr Cullen's paper illustrates the inherent impracticality of this design concept. It depends on a number of dimensional variables being initially correct and maintaining their relationship during operation. In addition, the sealing of the junction between the sill and the side seal becomes a 'hit or miss' arrangement. The effect of such a leakage under water is rarely observed but is often considerable.

Applying the experience of the portal seals at tunnel gates would result in a more effective arrangement, as shown in Fig. 9. Since dock gates close under no hydrostatic load, the gate seals would close unpressurised and therefore be relatively flexible. As the differential head builds up, the seal pressure increases.

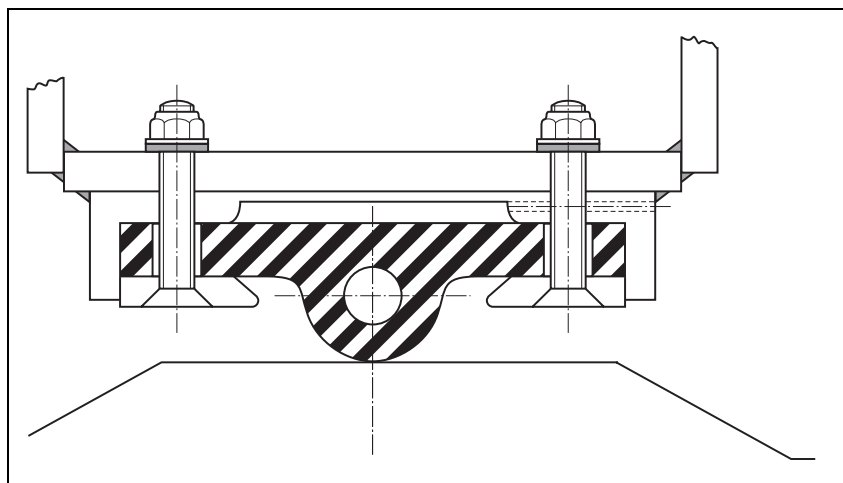

Fig. 9. Possible sill seal for a dock mitre gate
Another possible problem is the heal post bearing at the sill. Fig. 10 shows the design of the bearings of the mitre gates at Lower Gallions Reach on the tidal Thames. It is totally immersed in river mud as a consequence and bearing life is limited. The gate is operated only intermittently, otherwise it would be an intolerable arrangement. The use of a composite asbestos bearing under the operating conditions was an unfortunate choice. Self-lubrication bearings have the advantage of continuing to give good service under water, even if they become heavily scored.

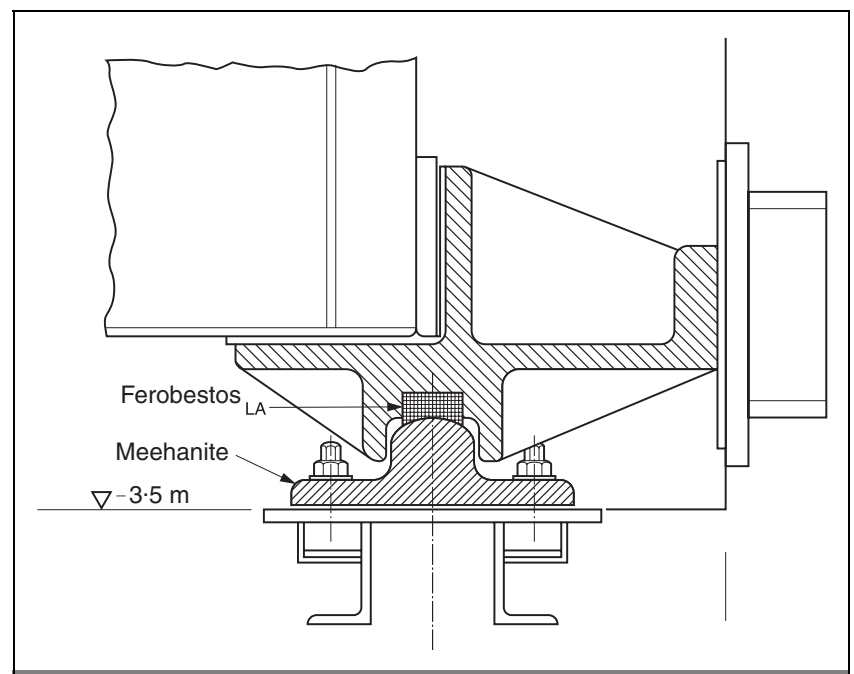

Fig. 10. Existing heal post bearing at a mitre gate

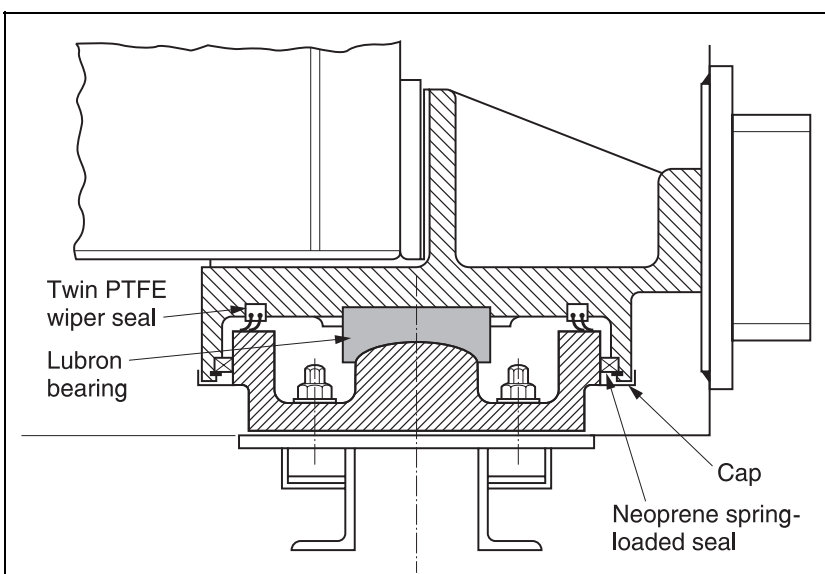

Fig. II. Improved heal post bearing for an existing mitre gate 
The arrangement of a heal post bearing, shown in Fig. 11, would be a substantial improvement. It is this contributor's opinion that wherever sedimentary matter can settle, heal post bearings should not be located at the sill and should take the form of hinge bearings.

\section{Author's reply}

There is no doubt the sill sealing arrangement of the majority of mitre gates is an unsatisfactory compromise of purpose and convenience leading inevitably to leaks. The sealing arrangement, as suggested, would produce a structural response more in line with the assumptions made in the traditional design process. However, for the suggested sealing system to be introduced to existing dock entrances there are two major obstructions that require consideration.

The existing sill and apron structure is, for most ports in the UK, permanently submerged under water to various depths. Access to the sill and apron to construct a surface for the gate seal to act on would require considerable financial investment due to the cost of de-watering. Installation could be achieved by the use of divers but due to a low level of visibility it would be unlikely an acceptable accuracy of level of the surface would be achieved. A solution to this obstruction requires a more in-depth study.

Operating equipment of most dock mitre gates is hydraulic cylinders and, with a few exceptions, the closing of each leaf of the gates is individually, manually controlled. With a lack of physical stop provided by the standing sill the mitre posts are less likely to meet in the 'middle' of the entrance. Such a situation would result in an unacceptable misalignment of the mitre posts, resulting in a concentration of stresses on the mitre posts and eccentricity on the gate structure. Control of engagement of the gate leaves could be achieved by use of appropriate mechanical/electronic devices. However, the freedom of the gate to strain would require an alternative to the traditional, flat-faced, timber mitre post as was suggested in paragraph 31 of the original paper.

Professor Lewin's second point does highlight siltation as a problem faced by many port operators. One effect of siltation is its abrasive action, which can accelerate wear on lower gudgeon/pintle bearing. While I welcome Professor Lewin's suggestion of an alternative scheme to protect the gudgeon/ pintle bearing I would be concerned as to the ease of engagement of the arrangement during the gate stepping. This activity is often carried out at high spring tide levels to make use of gate buoyancy to aid the manoeuvring and positioning of the gate. The use of divers in assisting the engagement of the hinge arrangement would have serious health and safety implications that generally exclude their involvement other than confirming the gate is in place after stepping is notionally complete. Installation of the gate in the dry, de-watered entrance could be financially excessive. Whether such a system of lower hinge is adopted, or not, would depend on the costbenefit analysis when the particulars of the gate (e.g. weight, tidal range, access for craneage) and the anticipated rate of accelerated wear on the bearing are considered.

As stated by Professor Lewin, there has been little development of traditional designs of mitre gates and I agree that development is long overdue. 